\title{
PERFORMANCE ANALYSIS OF DRAFT TUBE FOR GAMM FRANCIS TURBINE
}

\author{
T. KUBOTA \\ Kanagawa University \\ Yokohama, Japan
}

\author{
F. HAN \\ Huazhong University \\ Wuhan, China
}

\author{
F. AVELLAN \\ IMHEF, EPFL \\ Lausanne, Switzerland
}

\section{Introduction}

To precisely analyze the hydraulic losses in the various components of a Francis turbine, it is necessary to know the internal flow velocity distributions in the respective components. Since, however, the hydraulic energy loss $\Delta \psi$ and the performance characteristics such as energy coefficient $\psi$ and discharge coefficient $\phi$ etc. are all the one-dimensional information for a whole turbine, it is sufficient to know the sectional mean velocities along a single representative mid-streamline through the turbine. Thus, the one-dimensional flow theory is decisively useful for the loss analysis corresponding to the performance diagrams. Performance diagrams acquired by the precise model test are so reliable that utilizing the diagrams to the loss analysis is highly recommendable. Two of the authors have presented, applying the one-dimensional flow theory, a new algorithm of extracting the various component losses in bulb turbines from the performance diagrams measured with the model tests [1].

Hydraulic loss in a draft tube is strongly affected by the intensity of swirl flow at the runner outlet. Since the swirl intensity of runner outflow varies with the operating conditions of $\psi$ and $\phi$, the draft tube loss also depends on $\psi$ and $\phi$. So far, the effect of swirl intensity on the flow behavior in the draft tube is well-investigated relating to the pressure pulsations [2]. Little is reported, however, concerning its effect on the hydraulic energy loss in the draft tube. To analyze the draft tube losses versus $\psi$ and $\phi$ is essential to improve the hydraulic performance of Francis turbines, and to convert the model performance to its prototype considering the reliable scale effect.

GAMM Francis model turbine was designed and manufactured by IMHEF in EPFL to provide the experimental data on the hydraulic performances and flow distributions in the turbine for the 1989 GAMM Workshop on 3D-computation of incompressible internal flows [3]. The model test results revealed that the efficiency hill-diagram has the unusual two optimum peaks with the unsatisfactory efficiencies of 0.905 , respectively. To investigate the unusual performance of the turbine, a special test was added by relocating 
the low pressure measuring section for the determination of the specific hydraulic energy of turbine, SHET from the draft tube outlet to its inlet. The hill-diagram of the special test showed a single optimum peak with the improved efficiency of 0.920 [4].

The aim of this investigation is to extract the hydraulic energy loss in the bend draft tube for the GAMM Francis turbine from the above two performance diagrams, the one acquired by the normal model test with the draft tube outlet section, and the other by the special test with the draft tube inlet section.

\section{Specific Hydraulic Energy of Turbine SHET}

The specific hydraulic energy of turbine SHET shall, by nature, be defined as the difference of the true specific hydraulic energies between the inlet and outlet of the turbine. The true flow energy in a cross section can be determined by integrating the distributions of massaveraged pressures and kinetic energies including the swirl velocity under the assumption of the same potential energies between inlet and outlet. Practically, however, the IEC code stipulates to calculate SHET $E_{\text {nor }}$ with the following equation (cf. Fig. 1);

$$
E_{\text {nor }}=E_{C i}-E_{D o}=\left(\frac{P_{C i}}{\rho}+\frac{V_{C i}{ }^{2}}{2}\right)-\left(\frac{P_{D o}}{\rho}+\frac{V_{D o}{ }^{2}}{2}\right)
$$

where $P_{C l}$ and $V_{C i}$ is the wall pressure and the sectional mean velocity at the spiral case inlet, and $P_{D_{o}}$ and $V_{D_{o}}$ at the draft tube outlet, respectively. Equation (1) would be approximately true only when the flows at both the inlet and outlet sections are uniform without swirl. In general, when the flow has a swirl component, it is necessary to correct the swirl energy to the normal SHET $E_{\text {nor }}$ of Eq. (1).

Generally, a model test stand supplies rather uniform flow to the model turbine from the upper tank with rectifier via short straight inlet pipe. The flow in the high pressure measuring section is, therefore, nearly uniform without swirl irrespective of the operating conditions. The SHE (specific

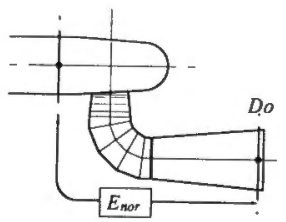

Fig. 1 Enor at normal test hydraulic energy) $E_{C l}$ at turbine inlet calculated with the wall pressure and the sectional mean velocity is approximately close to the true energy. On the other hand, the flow at runner outlet greatly varies with the operating conditions, and the bend draft tube distorts the flow toward the outlet. The flow at draft tube outlet is not uniform with strong distortion, swirl and/or reverse flows. As a result, the SHE $E_{D_{0}}$ at the draft tube outlet calculated with the wall pressure and the sectional mean velocity differs from the true energy. 
Under the operating condition of swirl-free flow at the runner outlet, the SHE $E_{D i}$ at draft tube inlet obtained from the wall pressure and the sectional mean velocity is rather close to the true energy. If we measure the special SHET $E_{s p c}$ by relocating the low pressure measuring section to the draft tube inlet as shown in Fig. 2 that differs from the IEC code specified, then, the SHET is close to the true energy at the swirl-free condition.

$$
E_{s p c}=E_{C i}-E_{D i}=\left(\frac{P_{C i}}{\rho}+\frac{V_{C i}{ }^{2}}{2}\right)-\left(\frac{P_{D i}}{\rho}+\frac{V_{D i}{ }^{2}}{2}\right)
$$

This $E_{s p c}$ reaches maximum at an operating point where the $E_{D i}$ becomes minimum under the given $E_{C i}$, and where the Euler energy of runner becomes maximum. Since $E_{D i}$

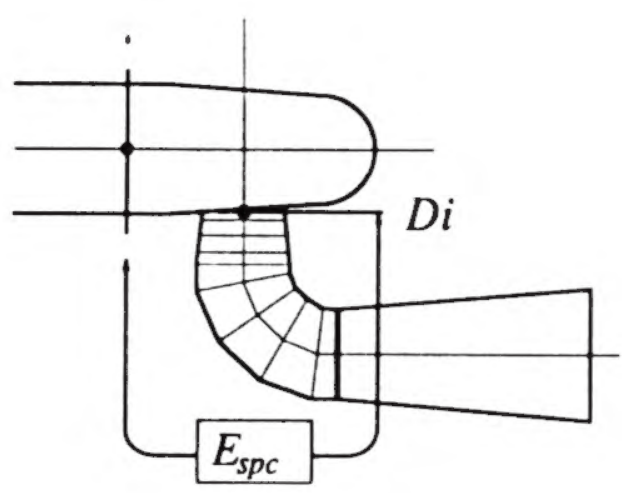

Fig. 2 Espc at special test reaches minimum at the point where the runner outflow has no swirl, the energy efficiency obtained by $E_{s p c}$ reaches maximum at this point. The special SHET $E_{s p c}$ does not include the hydraulic loss in the draft tube, so the efficiency based on $E_{s p c}$ must be higher than the one based on $E_{n o r}$. At the point where the runner outflow has swirl velocity, the swirl flow energy shall be corrected to both $E_{n o r}$ and $E_{s p c}$ for getting the true energy.

\section{Model Test Results}

\subsection{MODEL TURBINE AND TEST PROCEDURE}

The tested model Francis turbine has the discharge specific speed $n_{s Q}$ of 76 , the number of runner vanes $Z_{R} 14$, the runner outlet radius $R_{\text {ref }} 0.200 \mathrm{~m}$ and the runner vane outlet radius $R_{2}$ on the representative mid-streamline of $0.1394 \mathrm{~m}$. The adopted draft tube has an inlet conical diffuser with half cone angle $\theta_{D i}$ of $6.6 \mathrm{deg}$., a 90 deg.-bend of circular cross section with the constant area, an outlet conical diffuser with half cone angle $\theta_{D o}$ of 5.0 deg and the area ratio of outlet to inlet $A R$ of 3.23 .

The normal model test was executed by the experts of IMHEF by selecting the draft tube outlet to the low pressure measuring section according to the IEC code for the ten guide vane opening angles $\alpha_{G}$ from $15 \mathrm{deg}$. to $35 \mathrm{deg}$. The tested energy $E_{n o r}$ was set to be a constant value of $98 \mathrm{~J} / \mathrm{kg}$ according to Eq. (1).

After that, the special test followed by relocating the low pressure measuring section to the draft tube inlet with the same range of guide vane angles. The tested energy $E_{s p c}$ was also set to be a constant value of $98 \mathrm{~J} / \mathrm{kg}$ according to Eq. (2). 


\subsection{DIMENSIONLESS REPRESENTATION OF PERFORMANCES}

To find the corresponding operating points between the two tests, we need the dimensionless perfornances that are normalized to the identical reference conditions according to the similarity law. Since both the $E_{\text {nor }}$ and $E_{s p c}$ are adjusted to be the same value of $98 \mathrm{~J} / \mathrm{kg}$ during the tests, the actual energy for the special test is higher than the normal test, even under the same guide vane angle. Since the speed- and discharge-factor $n_{D E}$ and $Q_{D E}$ etc. based on the SHET $E$ can not be used, therefore, discharge-, torque- and energy-coefficient $\phi, \tau$ and $\psi$ must be adopted based on the peripheral speed at runner outlet $U_{\text {ref }}\left(=\omega R_{\text {ref }}\right)$ as follows;

$$
\begin{gathered}
\phi=\frac{Q}{\pi \omega R_{\text {ref }}{ }^{3}} \\
\tau=\frac{2 T}{\rho \pi \omega^{2} R_{\text {ref }}{ }^{5}} \\
\psi=\frac{2 E}{\omega^{2} R_{\text {ref }}{ }^{2}}
\end{gathered}
$$

The above $\phi$ and $\tau$ do not include $E$, so if the complete similarity to the internal flow through the model turbine is kept between the nomnal and the special test, the relation of $\tau$ versus $\phi$ shall coincide between the two tests irrespective of the tested energy $E$. As an example, Fig. 3 shows the correlation of $\tau$ and $\psi$ versus $\phi$ under the guide vane angle of $30 \mathrm{deg}$. The blank marks illustrate the normal test results and the filled marks correspond to the special test results. The torque coefficients for the both tests well coincide against the discharge coefficients and demonstrate the dimensionless performances of Eqs. (3) to (5) are applicable.

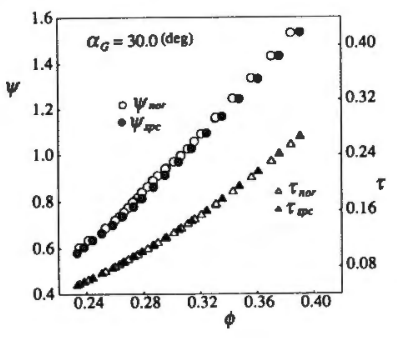

Fig. 3 Dimensionless Performances ( $\alpha_{G}=30$ deg.)

When the slight deviation is detected in the setting of $\alpha_{G}$ between the two tests that are executed separately, the value of $\phi$ is fine tuned to hold the consistency of $\phi$ versus $\alpha_{G}$ for both tests. The corresponding $\tau$ and $\psi$ are corrected with the square of $\phi$ by confirming the coincidence of $\tau$ and $\phi$. From the difference in $\psi$ thus corrected, now we can get the specific hydraulic energy of draft tube SHED $E_{s p c} E_{\text {nor }}$ 


\subsection{NORMAL TEST RESULTS}

The diagram of normal performances measured with $E_{n o r}$ are shown in Fig. 4. Strangely, the diagram has two efficiency peaks with the insufficient values of 0.905 under the lower discharge $\left(\phi_{\text {opt } 1}=0.26\right.$, $\alpha_{\text {Gopt } 1}=22.5 \mathrm{deg}$.) and the higher discharge $\left(\phi_{\text {opt } 2}=0.31, \alpha_{\text {Gopt2 }}=25\right.$

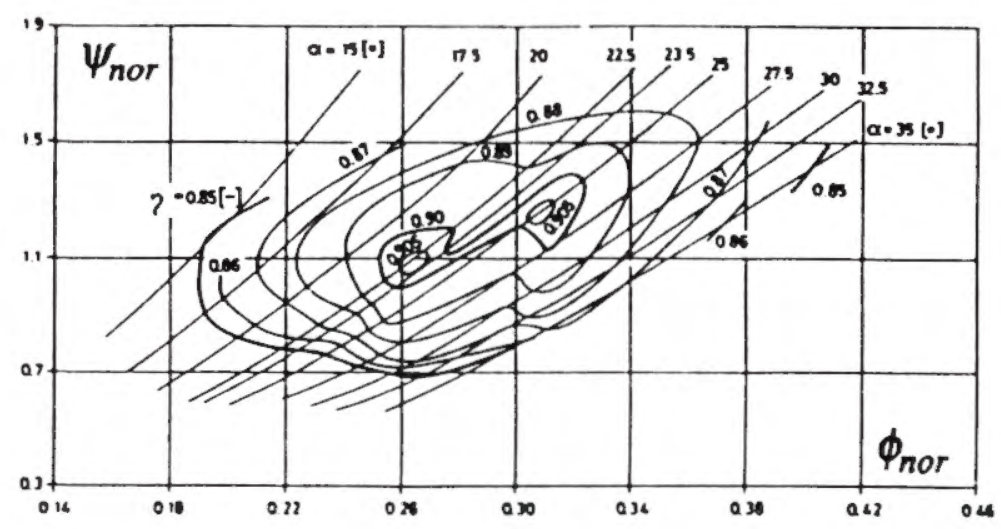
deg.).

Fig. 4 Normal Performances based on $E_{n o r}$

\subsection{SPECIAL TEST RESULTS}

The diagram of special performances measured with $E_{s p c}$ are shown in Fig. 5. The diagram has an improved single efficiency peak of 0.92 at the discharge coefficient of 0.29 . As mentioned above, the efficiency based on $E_{s p c}$ reaches optimum at the operating condition of swirl-free runner outflow. We can designate the optimum discharge in the special performances as the swirl-free discharge $\phi_{c f}(=0.29)$.

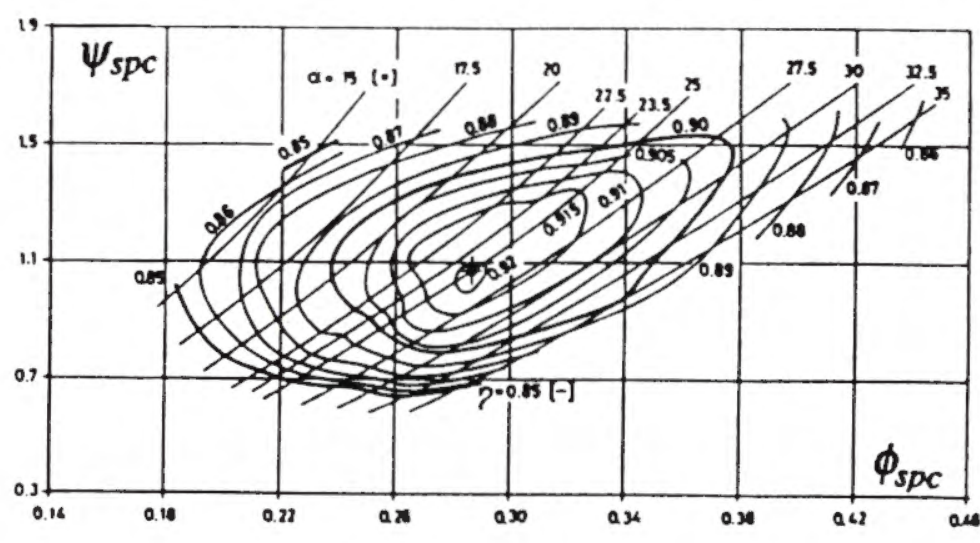

Fig. 5 Special Performances based on $E_{s p c}$

\section{Extraction of Draft Tube Energy}

As confirmed with Fig. 3, the difference in $\psi$ between the two performances is very small. To increase the sensitivity of detecting the small difference $\Delta \psi$ on the diagram, it is better to represent the diagram so as that the variation of ordinate is minimum against the variation of abscissa. For this reason, the energy $\psi$ and the torque $\tau$ of the two performances are transformed by multiplying the square and the cube of the ratio of the swirl-free discharge $\phi_{c f}$ to the respective discharge $\phi$, respectively. As the typical examples, Fig. 6 illustrates the magnified ordinates $\psi\left(\phi_{c f} / \phi\right)^{2}$ and $\tau\left(\phi_{c f} / \phi\right)^{3}$ versus $\phi$ for the guide vane angles of 25, 30 and $35 \mathrm{deg}$., respectively. Blank and filled mark corresponds to the 
normal and special performance, respectively. Both the $\tau_{\text {nor }}$ and $\tau_{s p c}$ are coincide well within a wide range of $\phi$ irrespective of $\alpha_{G}$. The magnified $\psi_{\text {nor }}$ varies much against $\phi$ with increasing $\alpha_{G}$, whereas the variation of $\psi_{s p c}$ is moderate.

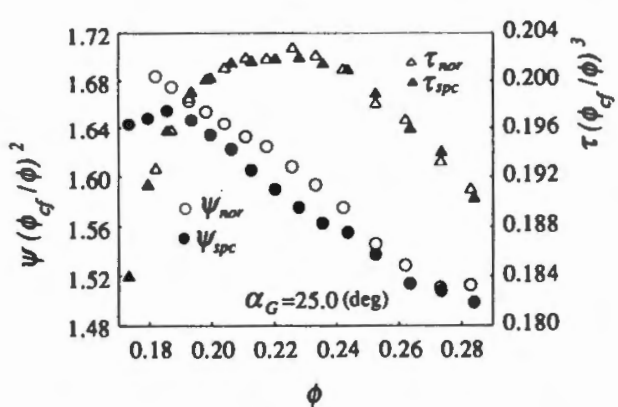

(a). $\alpha_{G}=25$ deg.

(c). $\alpha_{G}=35 \mathrm{deg}$.

Fig. 6 Magnified performances

The differential energy coefficient $\psi_{\text {nor }}$ $\psi_{\text {spc }}$ under the identical discharge coefficient $\phi$ implies the specific hydraulic energy of draft tube SHED based on the IEC definition

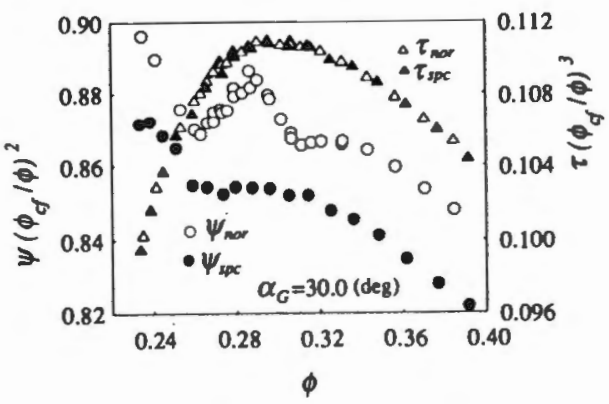

(b). $\alpha_{G}=30 \mathrm{deg}$.

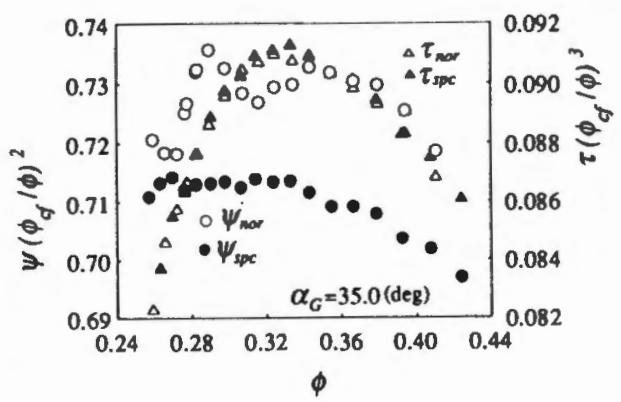

(wall pressures and sectional mean velocities) as follows;

$$
\Delta \psi_{I E C} \equiv \psi_{\text {nor }}-\psi_{s p c}=\frac{2\left(E_{D i}-E_{D o}\right)}{\omega^{2} R_{r e f}^{2}}
$$

The SHED thus extracted from the both performance diagrams are illustrated in Fig. 7 with the entire guide vane angles. The curves of $\Delta \psi_{I E C}$ repeat up and down against $\phi$ irrespective of $\alpha_{G}$ except the minimum angle of $15 \mathrm{deg}$. The local maxima on the middle of curves appear at the swirl-free discharge $\phi_{c f}$ of 0.29 irrespective of $\alpha_{G}$. According to the typical Fig. 6(b), this results from $\psi_{\text {nor }}$ including the draft tube loss and not from $\psi_{s p c}$. So, the local maxima is the evidence of sudden increase in draft tube energy necessary for the swirl-free flow at runner outlet.

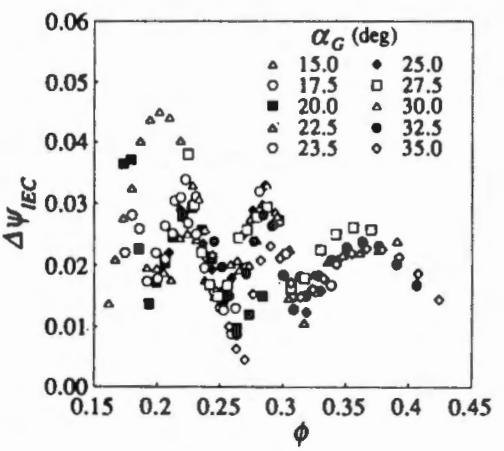

Fig. 7 Specific hydraulic energy of draft tube $\Delta \psi_{I E C}$ 


\section{Correction of Swirl Flow at Draft Tube Inlet}

As understood by Eq. (6), $\Delta \psi_{I E C}$ does not include the swirl energy at draft tube inlet. Actually, a Francis runner generates the swirl flow at runner outlet except the swirl-free operating conditions. The total kinetic energy of the swirl flow shall be the axial energy based on the sectional mean velocity plus the swirl energy. Also, the measured wall pressure includes the pressure rise due to the centrifugal force of swirl flow in addition to the static pressure. To obtain the actual energy at draft tube inlet, we have to correct the above effects of swirl flow as follows:

\subsection{SWIRL VELOCITY AT RUNNER OUTLET}

The velocity triangle at the intersection of a representative mid-streamline with the trailing edge line of runner vanes (subscript 2) forms the right triangle at the swirl-free discharge $\phi_{c f}$. The relative angle $\beta_{2 f f}$ of runner outflow can directly be obtained from $\phi_{c f}$ as follows;

$$
\tan \beta_{2 f} \equiv \frac{V_{m 2 f}}{U_{2 f}}=\phi_{c f}\left(\frac{\pi R_{r e f}^{3}}{A_{2} R_{2}}\right)
$$

where $A_{2}$ is the cross sectional area at runner vane trailing edge. The fact that the swirlfree discharge $\phi_{\mathrm{ef}}$ does not depend on the guide vane angle as shown in Fig. 7, tells us that also the runner outflow angle $\beta_{2 c j}$ is not dependent on the guide vane angle. This is understandable because the solidity of Francis runner vanes is high and, in general, the runer outflow angle is approximately constant within the normal operating range.

Assuming the runner outflow angle $\beta_{2}$ is constant $\left(=\beta_{2 c f}\right)$ irrespective of $\phi$. the swirl velocity $V_{u 2}$ of the ninner outflow can be calculated for the arbitrary $\phi$ as follows;

$$
V_{\mathrm{u} 2}=U_{2}-\frac{V_{m 2}}{\tan \beta_{2 f} \cdot}=U_{2}\left(1-\frac{\phi}{\phi_{f f}}\right)
$$

\subsection{SWIRL ENERGY AT DRAFT TUBE INLET}

The swirl energy coefficient $\Delta \psi_{v t}$ of the runner outflow can be obtained with the swirl velocity of Eq. (8) for the correction of the draft tube inlet energy as follows;

$$
\Delta \psi_{v u} \equiv \frac{V_{u 2}^{2} / 2}{U_{r e f}^{2} / 2}=\left(1-\frac{\phi}{\phi_{f f}}\right)^{2}\left(\frac{R_{2}}{R_{r f f}}\right)^{2}
$$

\subsection{WALL PRESSURE RISE DUT TO SWIRL FLOW}

Assuming the flow field between the runner vane trailing edge (position 2) and the low 
pressure measuring section at draft tube inlet (for $P_{D i}$ ) is of free-vortex, the wall pressure rise coefficient $\Delta \psi_{p}$ due to the swirl velocity of Eq. (8) is calculated with the following equation for the low pressure measuring section;

$$
\Delta \psi_{p} \equiv \frac{\Delta p / \rho}{U_{r e f}^{2} / 2}=\Delta \psi_{v u}\left(\frac{R_{2}}{R_{r e f}}\right)^{2}
$$

\section{Extraction of Draft Tube Loss}

By correcting the swirl energy $\Delta \psi_{v u}$ of Eq. (9) and the wall pressure rise $\Delta \psi_{p}$ of Eq. (10) to the IEC draft tube energy $\triangle \psi_{I E C}$ of Eq. (6), the effect of swirl flow on the energy at draft tube inlet has been corrected. Theoretically, the similar correction would be necessary for the swirl flow at draft tube outlet. As mentioned in Chap. 2, however, the flow at draft tube outlet is distorted greatly with the complicated swirl and reverse flow. The maximum velocity in the outlet section with swirl component would be much higher than the sectional mean velocity. Nevertheless, the kinetic energy of the tube outflow is negligibly small than the runner peripheral speed energy, except the case of high specific speed machines. The wall pressure at tube outlet is close to the pressure in the tail water tank, and less affected by the pressure rise because the swirl flow is not dominant. According to the above consideration, the actual energy loss $\Delta \psi_{D}$ in the draft tube flow can be to calculated with the following formula;

$$
\begin{aligned}
\Delta \psi_{D} & \equiv \frac{2 \Delta E_{D}}{\omega^{2} R_{r e f}^{2}}=\Delta \psi_{I E C}+\Delta \psi_{v u}-\Delta \psi_{p} \\
& =\Delta \psi_{I E C}+\left(1-\frac{\phi}{\phi_{c f}}\right)^{2}\left(\frac{R_{2}}{R_{r e f}}\right)^{2}\left(1-\frac{R_{2}^{2}}{R_{r e f}^{2}}\right)
\end{aligned}
$$

Figure 8 shows the actual draft tube loss $\Delta \psi_{D}$ obtained from SHED $\Delta \psi_{I E C}$ in Fig. 7 by using Eq. (11). Roughly speaking, the extracted draft tube loss coefficients are aligned on a single.line of W-shape except the minimum guide vane angle. The tube loss reaches maximum at the swirl-free discharge, and decreases once when the discharge leaves $\phi_{c f}$. As understood by Eq. (8), when the discharge decreases from the swirl-free discharge, the positive swirl velocity increases, and vice versa. There are two optimum discharges $\phi_{o p t}$ of 0.26 and 0.31

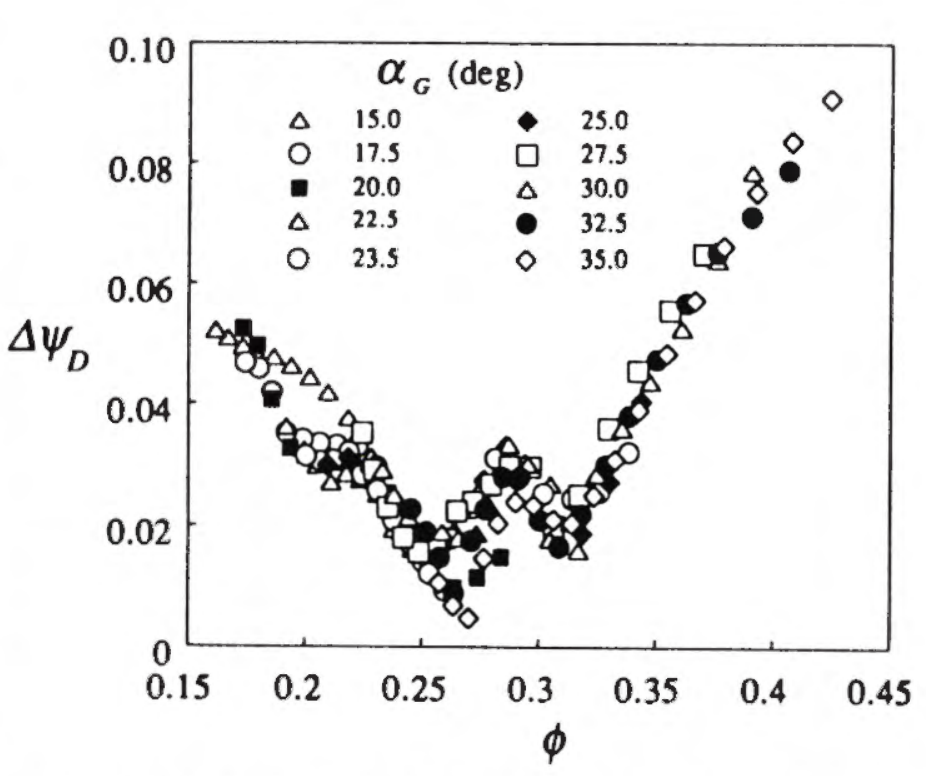

Fig. 8 Actual draft tube energy loss $\Delta \psi_{D}$ 
where the tube loss reaches minimum. The discharges can be designated as the optimum swirl discharge. The tube loss at the lower side of $\phi_{\text {opt }}$ is smaller than the loss at the higher side. At the lower side, the swirl energy at runner outflow is positively optimum, and vice versa. When the discharge decreases or increases apart from the optimum swirl discharges, the tube loss increases especially at high discharge range.

In general, if there is no-swirl flow at the inlet of a bend draft tube, the flow is used to separate from the inner bend wall, and the energy loss increases. The draft tube applied for this study has a circular bend of constant sectional area. The aspect ratio of the bend (the ratio of the radius of curvature at outer bend wall to that at inner bend wall) is high, and the secondary flow is apt to develop in the bend. This is the reason why the draft tube loss at the swirl-free discharge is large compared with the normal bend draft tube. The swirl energy at tube inlet increases when the discharge leaves the swirl-free discharge and suppresses the flow separation at the bend, resulting in the decrease of tube loss irrespective of the direction of swirl. Between the two minima of tube loss at the optimum swirl discharges, it is natural that the loss at the lower side of discharge is smaller. Since the tube loss at the swirl-free discharge is large, the contribution of swirl to the loss reduction is also large. Apart from the two optimum swirl discharges, with increasing positive or negative swirl energy, the tube loss becomes large.

Now we can understand the reason why the normal performance diagram has two efficiency peaks as follows: At the swirl-free discharge, the separation loss is large in the bend draft tube. At the two optimum swirl discharges smaller or larger than the swirl-free discharge, the separation loss is drastically suppressed by the optimum swirl. As a result, the two efficiency peaks appear at the two optimum swirl discharges. On the other hands, in the case of an ordinary Francis turbine with the well designed bend draft tube, since the separation loss in the bend is not so large, only a single efficiency peak will appear at the lower side of the two optimum swirl discharges.

The draft tube loss coefficient $\zeta_{D}$ based on the kinetic energy of the sectional mean velocity $V_{m D i}$ at draft tube inlet can be deduced from $\Delta \psi_{D}$ in Fig. 8 as follows;

$$
\zeta_{D} \equiv \frac{\Delta E_{D}}{V_{m D i}{ }^{2} / 2}=\frac{\Delta \psi_{D}}{\left(V_{m D i} / U_{r e f}\right)^{2}}
$$

The obtained results are illustrated in Fig. 9. The extracted draft tube loss coefficients are also aligned on a $\mathrm{W}$-shaped curve. The increase of coefficient is sharp at the lower discharge range, whereas the slope of curve is small at the higher discharge region with approaching to a constant value asymptotically.

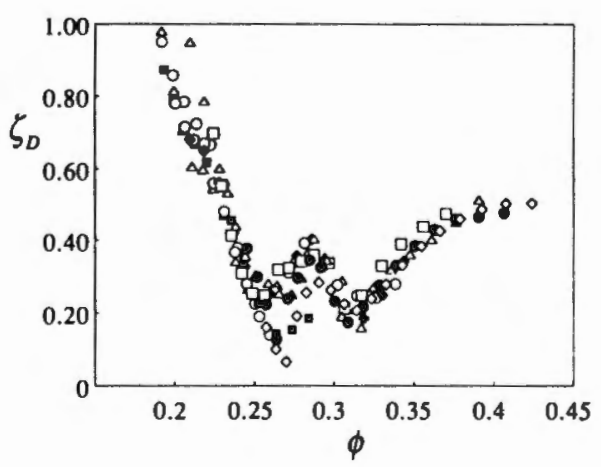

Fig. 9 Draft tube loss coefficient $\zeta_{D}$ 


\section{Conclusion}

Applying the GAMM Francis model turbine, the normal model test based on the draft tube outlet for the low pressure measuring section and the special test based on the draft tube inlet were executed separately. The SHED (specific hydraulic energy of draft tube) $\Delta \psi_{I E C}$ is extracted from SHET (specific hydraulic energy of turbine) for both performances under the identical flow conditions of the model turbines. By correcting the swirl energy at draft tube inlet to $\triangle \psi_{I E C}$, tne draft tube loss coefficient $\zeta_{D}$ can be empirically extracted from the both performance diagrams

\section{References}

1. Han, F., Ida, T. and Kubota, T.: Analysis of Scalable Loss in Bulb Turbine in Wide Operating Range, 17tn IAHR Symposium - Beijing (1994), Vol.2, G5, 853 - 864.

2. Kubota, T. and Yamada, S.: Effect of Cone Angle at Draft Tube Inlet on Hydraulic Characteristics of Francis Turbine, 11th IAHR Symposium - Amsterdam (1982), 53, 1-14.

3. Sottas, G. and Ryhming, 1.L.; "3D-Computation of Incompressible Internal Flows". Notes on Numerical Fluid Mechanics, Vol.39, (1993) Vieweg.

4. Avellan, F., Dupont, P., Farhat, M., Gindroz, B., Henry, P., Hussain, M., Parkison, E. and Santal, O.; "Flow Survey and Blade Pressure Measurements in A Francis Turbine Model", 15th IAHR Symposium - Belgrade (1990), Vol.2, 15. 